\title{
Additions and corrections of the family Mycetophagidae (Insecta: Coleoptera) from the Catalogue of Palaearctic Coleoptera, Volume 5 - Tenebrionoidea, 2020
}

\author{
JIǨÍ HÁVA
}

\author{
Forestry and Game Management Research Institute, \\ Strnady 136, CZ-252 02 Praha 5 - Zbraslav, Czech Republic \\ e-mail: jh.dermestidae@volny.cz; https://orcid.org/0000-0001-8076-9538
}

\begin{abstract}
Háva, J.: Additions and corrections of the family Mycetophagidae (Insecta: Coleoptera) from the Catalogue of Palaearctic Coleoptera, Volume 5 - Tenebrionoidea, 2020.

Abstract: Missing species, distributional data, references and corrections in the beetle family Mycetophagidae in the new Catalogue of Palaearctic Coleoptera, Volume 5, Tenebrionoidea are provided. The following species are newly recorded: Mycetophagus piceus Fabricius, 1777 (Nepal), M. quadriguttatus P.W.J. Müller, 1821 (Lebanon); Litargus balteatus LeConte, 1856 (Arab Emirates), Litargus connexus Geoffroy, 1785 (Israel), Triphyllus bicolor Fabricius, 1777 (Macedonia).
\end{abstract}

Keywords: additions, corrections, new records, catalogue, Coleoptera, Mycetophagidae, Palaearctic Region.

\section{Introduction}

The new Catalogue of Palaearctic Coleoptera. Tenebrionoidea. Revised and Updated Second Edition. Volume 5 edited by IwAN \& LöBL was published in 2020. The family Mycetophagidae in the new catalogue compiled by N.B. NiKITSKY. Some new species described in the years 2019 and 2020 (before publishing the catalogue), with new distributional data missing in the catalogue are added.

\section{Material and methods}

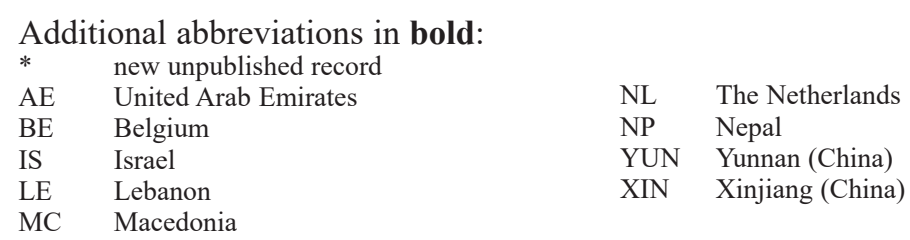


New references: HávA 2019a, b, 2020 are added.

Collections:

JHAC - Jiří Háva, Private Entomological Laboratory \& Collection, Únětice u Prahy, Prague-West, Czech Republic.

NMED - Naturkundemuseum, Erfurt, Germany.

PZAC - Petr Zahradník, private collection, Prague, Czech Republic.

\section{Results}

Family Mycetophagidae Leach, 1815

Page 27, additions: added one species and three distribution AE BE IS NL

Genus Eulagius Motschulsky, 1845

Eulagius filicornis (Reitter, 1887)c: 288 (Atritomus) E: BE FR GBi NL SP N: AG TU

Genus Litargus Erichson, 1846

Subgenus Alitargus Case, 1900

Litargus (Alitargus) balteatus LeConte, 1856: 14 E: AU AR CZ FR GB GE GG GR IR HU IR IT MA NL PL SK SL SP ST SV SZ N: CI EG MO MR A: AE JA AUR NAR NTR ORR PAC

Material examined: United Arab Emirates, Sharjah Desert Park, 25.17N 55.42E, at light trap, 6-30.4.2005, A v Harten lgt., 3 spec., J. Háva det., (JHAC). New to the United Arab Emirates.

Subgenus Litargus Erichson, 1846

Litargus (Litargus) connexus (Geoffroy, 1785): 138 (Antribus) E: AB AL AR AU BE BH BU BY CR CT CZ DE EN FI FR GB GE GG GR HU IR IT KZ LA LS LT MA MC MD NL NR NT PL PT RO SK SL SP ST SV SZ TR UK YU N: CI MO TU A: CH CY ES FE IN IS* JA JO KI KZ MG NC NE SY TR UZ

Material examined: Israel, Nizzanim, 18.vi.2009, L. Friedman leg., 1 spec., J. Háva det., (JHAC). New to Israel.

Subgenus Litargosomus Motschulsky, 1858

Litargus (Litargosomus) yunnanus Háva, 2020: 56 A: YUN

Page 28, additions: added one species and distribution NP 
Subgenus Ilendus Casey, 1900

Mycetophagus (Ilendus) yunnanus Háva, 2019b: 290 A: YUN

Subgenus Mycetophagoides Nikitsky, 1988

Mycetophagus (Mycetophagoides) alni Champion, 1917:53 A: NP UP

Page 29, additions: added two species and three distribution LE NL

Subgenus Mycetophagus Fabricius, 1792

Mycetophagus (Mycetophagus) ater (Reitter, 1879): 224 (Tritoma) E: AU BH BY CR CT CZ EN FI FR GE HU LA LT MD NL NT PL RO SK SL ST SZ UK YU A: CH ES FE JA KZ MG NC NE SC WS

Mycetophagus (Mycetophagus) weigeli Háva, 2020: 57 A: YUN

Subgenus Mycetoxides Motschulsky, 1858c

Mycetophagus (Mycetoxides) fulvicollis Fabricius, 1792b: 499 E: AB AL AR AU BE BH BU BY CR CT CZ DE EN FI FR GB GE GG GR HU IT KZ LA LT MD NL NR NT PL PT RO SK SL SP ST SV SZ UK YU A: ES FE KZ MG TR WS

Subgenus Parilendus Casey, 1900

Mycetophagus (Parilendus) quadriguttatus P.W.J. Müller, 1821: 198 E: AB AL AR AU BE BH BU BY CR CT CZ DE EN FI FR GB GE GG GR HU IT KZ LA LS LT MC MD NL NT PL RO SK SL SP ST SV SZ TR UK YU

N: AG MO A: IS KI KZ LE* SA SY TD TM TR UZ WS AUR NAR NTR ORR

Material examined: Lebanon, Rayfoun, ca. $33^{\circ} 58^{\prime} \mathrm{N}, 35^{\circ} 42^{\prime} \mathrm{E}$, mixed oak forest, 990 m, v.2015, pitfall trap, Reuter leg., 12 spec., J. Háva xdet., (10 NMED, 2 JHAC). New to Lebanon.

Subgenus Paramycetophagus Nikitsky, 2007

Mycetophagus (Paramycetophagus) nepalensis Háva, 2019a: 88 A: NP

Page 30, additions: added distribution NP

Subgenus Ulolendus Reitter, 1911

Mycetophagus (Ulolendus) piceus (Fabricius, 1777): 214 (Ips) E: AB AL AR AU BE BH BU BY CR CT CZ DE EN FI FR GB GE GG GR HU IT KZ LA LT LU MA MD NL NR NT PL PT RO SK SL SP ST SV SZ TR UK YU

N: EG A: KZ MG NP* TR UZ WS

Material examined: Nepal, P: Mahakali, D: Kanchanpur, vic. Mahendranagar, Hotel Suda N28 57'41'", E80¹2'51'", farm Sanctum, 230 m, LFF, 02.vii.2017, A. Weigel lgt., $1 \hat{\jmath}$, J. Háva det., (NMED). New to Nepal. 
Page 31, additions: added two distribution YUN, MC

Genus Typhaea Stephens, 1829

Typhaea stercorea (Linnaeus, 1758): 357 (Dermestes) E: AB AL AN AR AU AZ BE BH BU BY CR CT CZ DE EN FA FI FR GB GE GG GR HU IC IR IT KZ LA LS LT LU MA MC MD NL NR NT PL PT RO RU SK SL SP ST SV SZ TR UK YU N: AG CI EG LB MO MR TU A: AE AF BT CH CY ES FE IN IQ IS JA JO KI KZ MG NC NP PA SA SC SI SY TD TM TR UZ YUN WS YE COS

Genus Triphyllus Dejean, 1821

Triphyllus bicolor (Fabricius, 1777): 216 (Nitidula) E: AB AL AR AU BE BH BU BY CR CT CZ DE EN FI FR GB GE GG GR HU IT LA LT MC* MD NL NR NT PL PT RO SK SL SP ST SV SZ UK YU A: TR

Material examined: Macedonia, Galičica n. park, 3.6.1996, P. Zahradník lgt., J. Háva det., (PZAC). New to Macedonia.

\section{Page 32, corrections:}

angusta Rosenhauer, 1856 (Typhaea) - the species transferred by EsSER (2017) to the genus Cryptophilus Reitter, 1874 (Erotylidae) and removed it from the family Mycetophagidae. Nikitsky (2020) mentioned the species erroneously in Mycetophagidae.

\section{Acknowledgements}

The paper was supported by the Ministry of Agriculture of the Czech Republic, institutional support MZE-RO0118.

\section{References}

ESSER J. 2017: Was ist Typhaea angusta Rosenhauer, 1856 (Coleoptera)?. - Entomologische Nachrichten und Berichte 61(3-4): 177-179.

Háva J. 2019a: A new Mycetophagus Fabricius, 1792 species from Nepal (Coleoptera: Mycetophagidae). Acta Biologica Universitatis Daugavpiliensis 19(1): 87-90.

Háva J. 2019b: A new species of the genus Mycetophagus Fabricius, 1792 (Coleoptera: Mycetophagidae) from China. - Euroasian Entomological Journal 18(4): 290-291.

HÁva J. 2020: Two new species of the family Mycetophagidae (Coleoptera) from China. - Euroasian Entomological Journal 19(1): 56-58.

IwAN D. \& LöBL I. 2020: Catalogue of Palaearctic Coleoptera. Tenebrionoidea. Revised and Updated Second Edition. Volume 5. Leiden/Boston, Brill, 945 pp.

Nikitsky N.B. 2020: Mycetophagidae, Pp: 27-33. In: IwAn D. \& LöBl I. (eds.): Catalogue of Palaearctic Coleoptera. Tenebrionoidea. Revised and Updated Second Edition. Volume 5. Leiden/Boston, Brill, 945 pp. 